\title{
Synthesis of Mesoporous Material from Chrysotile-Derived Silica
}

\author{
Anderson Joel Schwanke ${ }^{1 *}$, Christian Wittee Lopes ${ }^{2}$, Sibele Berenice Castellã Pergher ${ }^{1,2}$ \\ ${ }^{1}$ PPGCEM, Universidade Federal do Rio Grande do Norte, Natal, Brazil; ${ }^{2}$ Chemistry Institute, Universidade Federal do Rio Grande \\ do Norte, Natal, Brazil. \\ Email: *anderson-js@live.com
}

Received June $15^{\text {th }}, 2013$; revised July $17^{\text {th }}, 2013$; accepted July $29^{\text {th }}, 2013$

Copyright (C) 2013 Anderson Joel Schwanke et al. This is an open access article distributed under the Creative Commons Attribution License, which permits unrestricted use, distribution, and reproduction in any medium, provided the original work is properly cited.

\begin{abstract}
Mesoporous MCM-41-type molecular sieves were synthesized using calcined and leached chrysotile and cetyltrimethylammonium bromide as the silica source and structure directing agent, respectively. Powder X-ray diffraction (XRD), $\mathrm{N}_{2}$ isothermal adsorption-desorption, scanning electron microscopy (SEM) and thermogravimetric analysis (TGA) were used to characterize the samples. The calcined and leached chrysotile can be employed as an inexpensive silica source for the formation of low-order MCM-41 mesoporous materials.
\end{abstract}

Keywords: MCM-41; Chrysotile; Silica Source

\section{Introduction}

Amiant and asbestos are generic names for fibrous minerals; serpentine group minerals are also included in this category, of which chrysotile is a member. The discovery of the world's third largest deposit of chrysotile in the beginning of the 1960s in the north of the Brazilian state of Goiás changed Brazilian participation in the world minerals market; with several applications in the modern world, amiant played a key role in the social and economic development of Brazil. Low-cost roofs and water tanks are manufactured with the amiant, allowing people easier access to habitation and basic sanitation. In addition to supplying internal market demand, Brazil exports amiant fibers to Latin American, Asian and African countries. Some studies reported that chrysotile fiber production in 2004 was as high as 250,000 tons [1], making increases in the value of this inexpensive and abundant material an attractive prospect.

In chemical terms, chrysotile is a hydrated lamellar silicate with a 1:1 structure, as shown in Figure 1. The chemical formula is $\mathrm{Mg}_{6}\left(\mathrm{Si}_{4} \mathrm{O}_{10}\right)(\mathrm{OH})_{8}$, though there can be some $\mathrm{Fe}^{+3}$ substitution into $\mathrm{Mg}^{+2}$ sites. Because of lamellar mismatch arising from different bond angles, chrysotile forms a wrapped fibrous structure with a silicate layer located inside a brucite layer [2].

\footnotetext{
${ }^{*}$ Corresponding author.
}

Some papers report the possibility of obtaining amorphous silica from chrysotile. This procedure is accomplished by subjecting chrysotile fibers to an acid treatment, allowing the removal of the outer brucite magnesium layer [3].

The use of chrysotile for the synthesis of porous materials is thus far relatively unexplored. A few studies report the synthesis of microporous materials such as ZSM-5 and $\mathrm{NaA}$ zeolites using natural chrysotile as a silica source [4-6]. Additionally, some studies report the use of silica from chrysotile in the production of nanofibers [7] and nanowires [8]. However, no studies on the synthesis of mesoporous materials such as MCM-41 using silica from natural chrysotile have been reported in the literature.

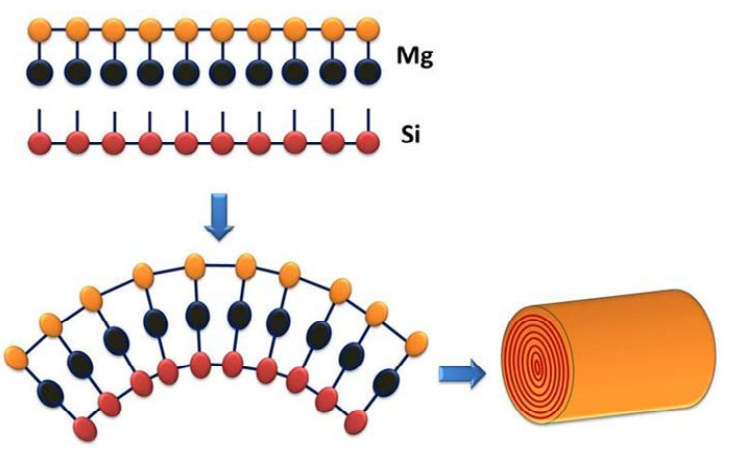

Figure 1. Chrysotile structure. 
In 1992, a major barrier was broken in nanostructured materials synthesis; the discovery of a novel family of materials called M41S, which exhibited the properties of mesoporous molecular sieves, spurred an increase in scientific research in this field [9]. MCM-41 belongs to this family and new applications and synthetic routes using alternative raw materials are reported often. The most relevant factors contributing to the interest in these materials are their high thermal stability, pore sizes ranging from 2 to $20 \mathrm{~nm}$, specific area of $\sim 700 \mathrm{~m}^{2} \cdot \mathrm{g}^{-1}$, morphological features and easy synthesis.

The majority of the applications for this material have been registered by the Mobil Oil Co. [10] and are related to cracking and hydrocracking of hydrocarbons. Nevertheless, this material may have major applications in the fields of catalysis and adsorption as well as more general applications related to the inability of bulky molecules to enter in the channels of microporous crystalline materials [11].

MCM-41 synthesis employs three ingredients: a solvent, which is usually basic; a siliceous source material, which can be any one of a number of alternative siliceous materials, such as rice husks [12], kaolin [13], and coal fly ash [14]; and the structure directional agent, a surfactant.

The purpose of this study is to synthesize nanostructured materials, such as MCM-41, from chrysotile-derived silica for possible future applications in adsorption and catalysis.

\section{Experimental}

\subsection{Obtaining Silica from Chrysotile}

Natural chrysotile from Goiás (Brazil) was heat treated with a heating ramp of $100^{\circ} \mathrm{C}$ per hour to $600^{\circ} \mathrm{C}$ and then held at this temperature for 3 hours. After cooling, the samples were treated with aqueous $\mathrm{HCl}\left(6 \mathrm{~mol} \cdot \mathrm{L}^{-1}\right)$ under reflux for 48 hours at $100^{\circ} \mathrm{C}$. The material was filtered with deionized water until a $\mathrm{pH}$ of 7 was reached and then dried at $100^{\circ} \mathrm{C}$ overnight.

\subsection{Synthesis of Mesoporous Material}

The obtained material was synthesized with the following molar ratio: $1 \mathrm{SiO}_{2}$ : 0.1 CTABr: 0.25 TMAOH: 20 $\mathrm{H}_{2} \mathrm{O}$.

The procedure was the same that described by Dr. Avelino Corma (Villalba, 1997) [15]. First, a reference sample was synthesized using commercial silica (Aerosil 200, Degussa); these samples were called SiMCM-4. Amorphous chrysotile-derived silica was then synthesized in an identical manner; these samples were called MSCris (Chrysotile Molecular Sieve).

Solution A: $5.00 \mathrm{~g}$ of hexadecyltrimethylammonium bromide-CTABr (Aldrich) was dissolved in $33.5 \mathrm{~g}$ of distilled water. Solution B: $8.65 \mathrm{~g}$ of $25 \%$ tetramethylammonium hydroxide-TMAOH (Aldrich) was dispersed on $0.96 \mathrm{~g}$ of silica.

Solution A was added to solution B. In addition, $4.52 \mathrm{~g}$ of silica was added and stirred for 1 hour. The resultant gel was transferred to a Teflon-lined autoclave and heated under autogenous pressure at $135^{\circ} \mathrm{C}$ for 24 hours without stirring. The material was filtered with deionized water and dried at $100^{\circ} \mathrm{C}$ overnight.

The obtained material was calcined at $550^{\circ} \mathrm{C}$ for 4 hours under nitrogen and synthetic air flow.

\subsection{Characterization}

The obtained materials were characterized by several techniques, namely, X-ray powder diffraction (XRD), scanning electron microscopy (SEM), nitrogen adsorption by the BET method and thermogravimetric analysis (TGA). The X-ray diffraction analyses were recorded on a Diffraktometer, model D5000 (Siemens) using a Ni filter and $\mathrm{Cu}-\mathrm{k} \alpha$ radiation under a $30 \mathrm{kV}$ accelerator voltage and $15 \mathrm{~mA}$ current. The nitrogen adsorption-desorption isotherms were measured using a Quanta ChromeNova 1000 series. Before analysis, the samples were degassed at $300^{\circ} \mathrm{C}$ during 12 hours in a vaccum furnace. The surface area is calculated using the BET equation [16]. The model of Barret, Joyner and Halenda (BJH) [17] were used for the mesoporose size distribuitions. For the scanning electron microscopy (SEM), samples were coated with a gold film and analyzed with a JOEL-JSM 5800 under a $20 \mathrm{kV}$ acceleration voltage. The thermogravimetric analysis (TGA) was performed in a Shima$\mathrm{dzu}$, TGA-50H with heating/cooling rates of $10^{\circ} \mathrm{C} \cdot \mathrm{min}^{-1}$ under synthetic air flow.

\section{Results and Discussion}

Figure 2 shows the X-ray diffractogram of natural chrysotile (a) and its calcined and leached form (b). The characteristic diffraction peaks of the chrysotile were observed at $\mathrm{d}=8.08$ and $4.03 \AA$, corresponding to the (002) and (004) planes respectively.

These results are consistent with the results reported in the literature [18]. A small amount of brucite is present in the structure by the presence of a peak at $2 \theta=18.60^{\circ}$, corresponding to the (001) reflection. In the calcined and leached chrysotile (b), a broad, low intensity peak is observed at $2 \theta=15^{\circ}$ to $30^{\circ}$, an indicator of the amorphous nature of this material. The absence of a corresponding chrysotile peak indicates that the calcination and acid treatment were effective. Figure 3 presents the XRD pattern of the samples synthesized with calcined and leached chrysotile (MSCris) and with commercial silica (SiMCM-41). The MSCris diffractogram has only one peak at $2 \theta=1.97^{\circ}$, corresponding to the (100) plane, 


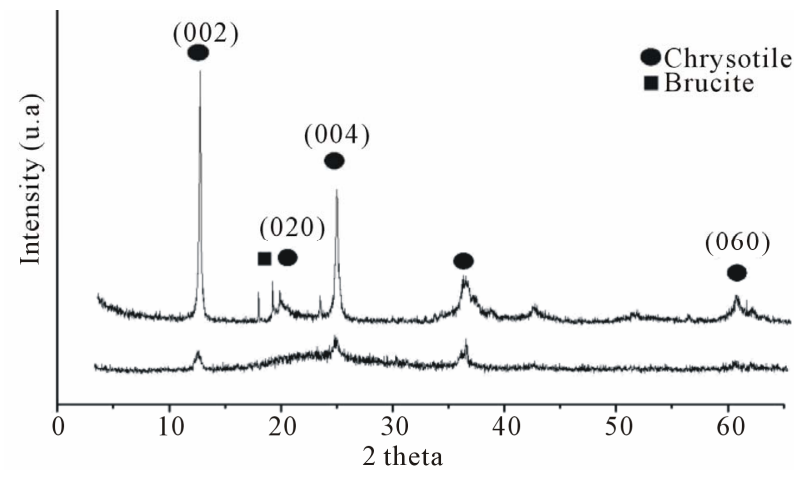

Figure 2. X-ray diffractogram of natural chrysotile (a) and calcined and leached chrysotile (b).

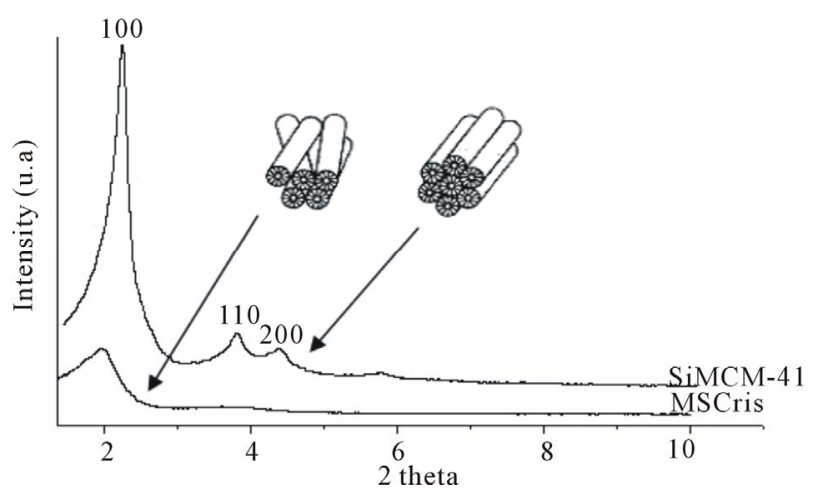

Figure 3. X-ray diffractogram of synthesized MSCris and reference SiMCM-41 samples.

which is characteristic of a hexagonal pore system. The absence of peaks for (110) and (200) reflections indicate that the material has a disoriented unidirectional structure, as previously described in the literature [19]. In the SiMCM-41 reference, three characteristic peaks from MCM-41 are observed. The material was furthermore shown to have good thermal stability because no structural degradation was observed when the samples were submitted to calcination temperatures above $500^{\circ} \mathrm{C}$.

The BET specific area analysis nitrogen adsorption iso- therms for the MSCris and SiMCM-41 samples are shown in Figure 4.

The high specific areas $\left(698\right.$ and $1090 \mathrm{~m}^{2} \cdot \mathrm{g}^{-1}$ for the MSCris and SiMCM-41 samples, respectively) and type IV isotherms without microporous phases demonstrate the mesoporous characteristics of the MSCris and SiMCM-41 materials. Pore size distributions calculated by the BJH method for the MSCris and SiMCM-41 samples with peak values near 32.7 and $31.6 \AA$ for the MSCris and SIMCM-41 samples, respectively. Both samples possess a mesoporous structural order. As shown by the similarity of the pore diameter distributions, the synthesized particles of MCM-41 material are not significantly affected by the source of the silica but are instead largely affected by the size of the alkyl chain of the surfactant molecules, as previously reported in the litera- ture [20].

An SEM micrograph of calcined and leached chrysotile is show in Figure 5, indicating the loss of its fibrous structure, as previously reported by Petkowicz [4].

The SEM micrograph of MSCris in Figure 6(a) shows clusters with irregular morphology and sizes greater than $50 \mu \mathrm{m}$.

The fibrous form of some particles indicates the presence of incompletely dissolved chrysotile, suggesting a possibly incomplete thermal and chemical treatment. The SEM micrograph of the SiMCM-41 reference sample shown in Figure 6(b) shows some $50 \mu \mathrm{m}$ particles with a large quantity of $7 \mu \mathrm{m}$ particles. This is a better distribution compared with the MSCris sample. This result can be explained by the greater reactivity of the commercial Aerosil 200 silica compared with the chrysotile-derived silica.

Figure 7 shows the thermogravimetric analysis (TGA) results of MSCris and SiMCM. According to the literature [21], MCM-41 has three mass loss features. At temperatures between 25 and $150^{\circ} \mathrm{C}$, desorption of physically adsorbed water occurs, resulting in mass losses of approximately $5 \%$ in both samples. At temperatures between 150 and $400^{\circ} \mathrm{C}$, the decomposition of the hexagonally arranged surfactant occurs. At temperatures above $400^{\circ} \mathrm{C}$, mass loss due to dehydroxylation of silanol groups present on the network occurs. The two synthesized samples, MSCris and SiMCM-41, have similar mass loss profiles.
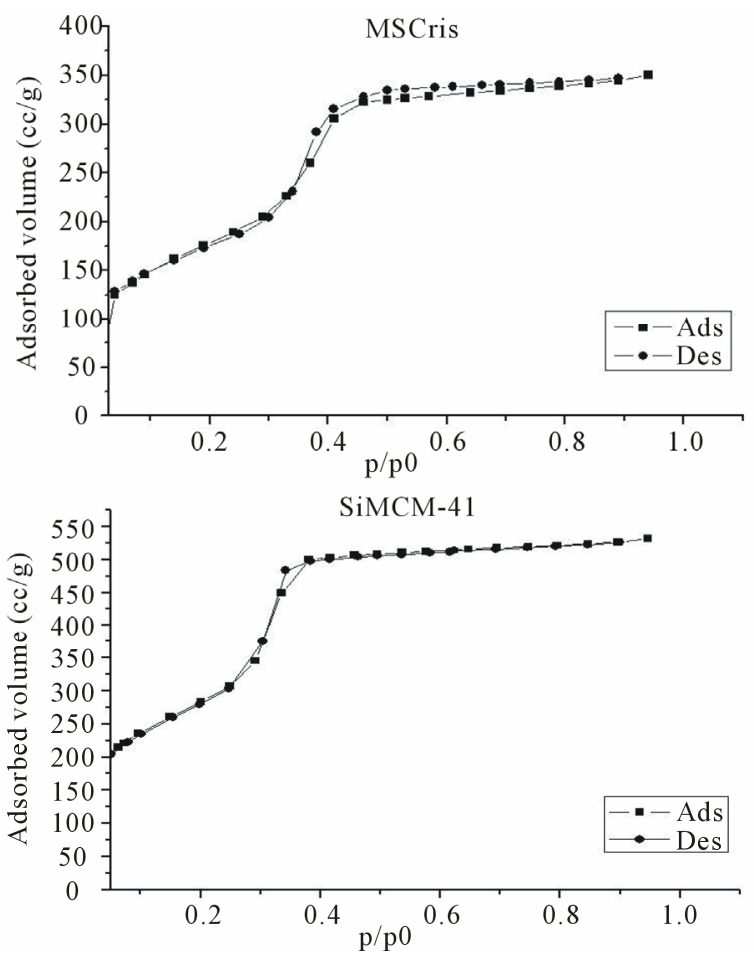

Figure 4. Nitrogen adsorption-desorption isotherms of MSCris and reference SiMCM-41 samples. 


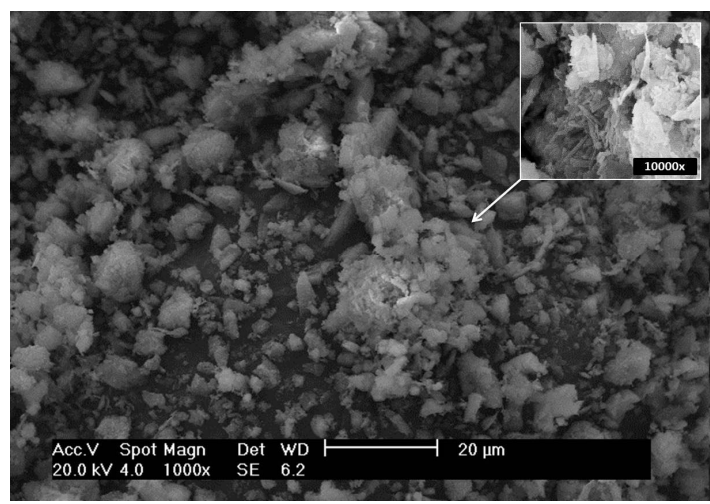

Figure 5. Scanning electron microscope micrograph of calcined and leached chrysotile sample at $1000 \times$ magnification.

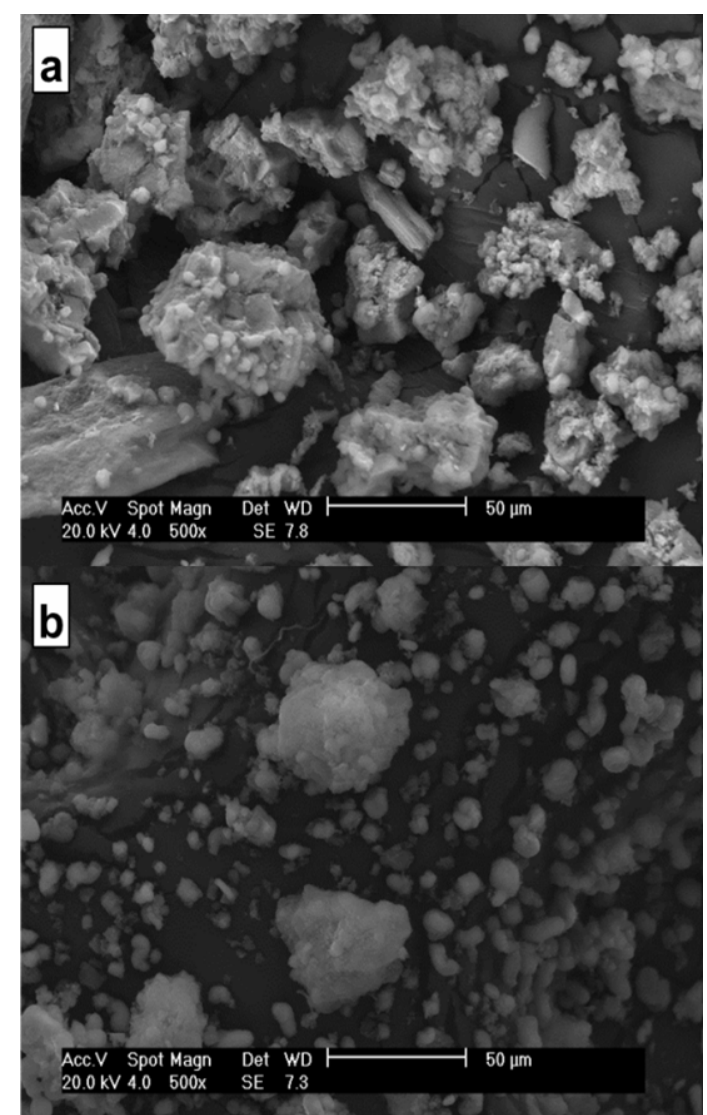

Figure 6. Scanning electron microscope micrographs of (a) MSCris and (b) SiMCM-41 samples at 500× magnification.

\section{Conclusion}

The synthesis of a nanostructured material from chrysotile-derived silica was carried out successfully. The analysis of specific surface area indicates that the material is mesoporous. Nitrogen adsorption-desorption isotherms present the type IV characteristic regions of mesoporous materials. The pore size distribution observed also fits that of nanoscale mesoporous materials. Thermogravimetric analysis shows that the MSCris sam-

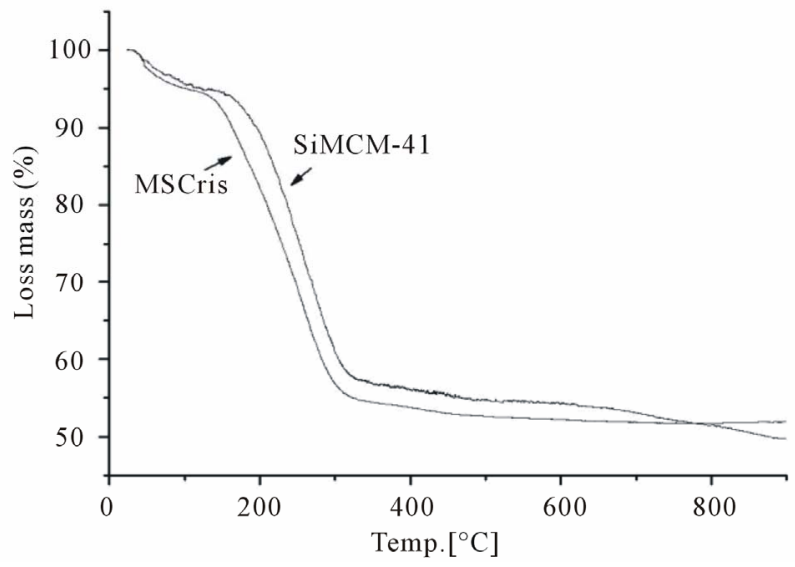

Figure 7. Thermogravimetric analysis of MSCris and reference SiMCM-41 samples.

ple has a similar mass loss profile to that of the SiMCM41 reference sample.

\section{Acknowledgements}

The authors gratefully acknowledge financial support from CAPES organization, as well as the NEPGN-UFRN organization for use of their Scanning Electron Microscopy.

\section{REFERENCES}

[1] Mineral Commodity Summaries, 2005. http://www.crisotilabrasil.org.br/site/pesquisas/_.../Relato rio $\% 20$ Instituto\%20.pdf

[2] D. M. Bernstein and J. A. Hoskins, "The Health Effects of Chrysotile: Current Perspective Based upon Recent Data," Toxicology and Pharmacology, Vol. 45, No. 3, 2006, pp. 252-264. doi:10.1016/j.yrtph.2006.04.008

[3] A. Hargreaves and W. H. Taylor, “An X-Ray Examination of Decomposition Products of Chrysotile (Asbestos) and Serpentine," Mineralogical Magazine, Vol. 27, 1946, p. 204. doi:10.1180/minmag.1946.27.193.05

[4] D. I. Petkowicz, R. T. Rigo, C. Ratdke, S. B. C. Pergher and J. H. Z. Santos, "Zeolite NaA from Brazilian Chrysotile and Rice Husk," Microporous and Mesoporous Materials, Vol. 116, No. 1-3, 2008, pp. 548-554. doi:10.1016/j.micromeso.2008.05.014

[5] P. Levesque, D. Bianchi, L. V. Mao and G. M. Pajonk, "Effect of Magnesium in the Conversion of Methanol on Chryso-Zeolite or Zeolite ZSM-5 Catalysts," Applied Catalysis, Vol. 57, No. 1, 1990, pp. 31-43. doi:10.1016/S0166-9834(00)80721-6

[6] R. Le Van Mao, P. Kipkemboi, P. Levesque, A. Vaillancourt and G. Denes, "Asbestos-Derived Zeolites as WaterRetaining Materials in Soils," Zeolites, Vol. 11, No. 8, 1991, pp. 804-809. doi:10.1016/S0144-2449(05)80059-4

[7] L. Wang, A. Lu, C. Wang, Z. Zheng, D. Zhao and R. Liu, "Nano-Fibriform Production of Silica from Natural Chrysotile," Journal of Colloid and Interface Science, Vol. 
295, No. 2, 2006, pp. 436-439. doi:10.1016/j.jcis.2005.08.055

[8] K. Liu, Q. Feng, Y. Yang, G. Zhang, L. Ou and Y. Lu, "Preparation and Characterization of Amorphous Silica Nanowires from Natural Chrysotile," Journal of NonCrystalline Solids, Vol. 353, No. 16-17, 2007, pp. 15341539. doi:10.1016/j.jnoncrysol.2007.01.033

[9] B. Sophie and L. M. Ocelli, "Synthesis and Characterization of Mesostructured Materials," Catalysis Reviews, Science and Engineering, Vol. 40, No. 3, 1998, p. 399.

[10] C. T. Kresg, M. E. Leonowicz, W. J. Roth, J. C. Vartuli and J. S. Beck, "Ordered Mesoporous Molecular Sieves Synthesized by a Liquid-Crystal Template Mechanism," Nature, Vol. 359, 1992, p. 710. doi:10.1038/359710a0

[11] J. Cejka and H. van Bekkum, "Zeolites and Ordered Mesoporous Materials: Progress and Prospects," Studies in Surface Science and Catalysis, Vol. 157, No. 9, 2005, p. 394.

[12] S. Chiarakorna, T. Areeroba and N. Grisdanurak, "Influence of Functional Silanes on Hydrophobicity of MCM41 Synthesized from Rice Husk," "Science and Technology of Advanced Materials, Vol. 8, No. 1-2, 2007, pp. 110-115. doi:10.1016/j.stam.2006.11.011

[13] F. Kang, Q. Wang and S. Xiang, "Synthesis of Mesoporous Al-MCM-41 Materials Using Metakaolin as Aluminum Source," Materials Letters, Vol. 59, No. 11, 2005, pp. 1426-1429. doi:10.1016/j.matlet.2004.11.057

[14] K. S. Hui and C. Y. H. Chao, "Synthesis of MCM-41 from Coal Fly Ash by a Green Approach: Influence of Synthesis pH," Journal of Hazardous Materials, Vol. 137, No. 2,
2006, pp. 1135-1148. doi:10.1016/j.jhazmat.2006.03.050

[15] M. T. N. Villalba, "Synthesis, Characterization and Catalytic Activity of MCM-41 Mesoporous Materials," Thesis, Valencia Polytechnic University, 1997, p. 265.

[16] S. Brunauer, P. H. Emmett and E. Teller, "Gases in Multimolecular Layers," Journal of the American Chemical Society, Vol. 60, No. 1, 1938, pp. 309-419. doi:10.1021/ja01269a023

[17] E. P. Barret, L. G. Joyner and P. P. Halenda, "The Determination of Pore Volume and Area Distributions in Porous Substances. I. Computations from Nitrogen Isotherms," Journal of the American Chemical Society, Vol. 73, No. 1, 1951, pp. 373-380. doi:10.1021/ja01145a126

[18] H. Yang, Y. Xiao, K. Liu, Y. Yang and Q. Feng, "Physicochemical Dispersion of Chrysotile," Colloids and Surfaces A: Physicochemical and Engineering Aspects, Vol. 301, No. 1-3, 2007, pp. 341-345. doi:10.1016/j.colsurfa.2006.12.071

[19] C. Y. Chen, S. Q. Xiao and M. E. Davis, "Studies on Ordered Mesoporous Materials III. Comparison of MCM41 to Mesoporous Materials Derived from Kanemite," Microporous Materials, Vol. 4, No. 1, 1995, pp. 1-20.

[20] C. Siriluk and S. Yuttapong, "Structure of Mesoporous MCM-41 Prepared from Rice Husk Ash," The 8th Asian Symposium on Visualization, Chaingmai, 23-27 May 2005, pp. 23-27.

[21] C. Y. Chen, H. X. Li and M. E. Davis, "Studies on Mesoporous Materials: I. Synthesis and Characterization of MCM-41," Microporous Materials, Vol. 2, No. 1, 1993, pp. 17-26. 